\title{
Percepción Social de la Violencia en la Pareja desde los Estereotipos de Género
}

\section{Social Perception through Gender Stereotypes of Partner Violence}

\author{
Leonor M. Cantera y Josep M. Blanch \\ Universidad Autónoma de Barcelona
}

\begin{abstract}
Resumen. El objetivo general de la investigación consiste en evaluar el grado de anclaje social de determinados estereotipos sobre género (hombre proveedor; mujer cuidadora) y sobre violencia de género (hombre violento, mujer pacífica) y se enmarca en el contexto de un debate sobre el alcance y los límites del enfoque de género a la hora de comprender y prevenir la violencia en las diversas modalidades de pareja. Participaron en la investigación 741 personas, dos tercios de las cuales mujeres, residentes en España, México, Puerto Rico y El Salvador. En cada país, se accedió a una muestra de conveniencia estratificada de acuerdo con criterios de género, generación, nivel de formación, situación ocupacional y orientación sexual. En sesiones individualizadas cuya duración osciló entre 35 y 60 minutos, las personas participantes respondieron primero un IAT (Implicit Association Test) y luego un cuestionario con series de ítems cerrados y de preguntas abiertas. Una de sus secciones incluye 48 ítems referidos a "actividades" que la persona debe categorizar numéricamente en una escala de 1 a 7 , con formato de diferencial semántico, en cuyos polos figuran "de hombre” y "de mujer”. En esta serie se entremezclan dos escalas de 24 ítems cada una de ellas: la de dureza y la de ternura. De la información obtenida se desprende que las muestras de todos los países organizan su percepción de la violencia en la pareja de acuerdo con los estereotipos de género. Hombres y mujeres coinciden en percibir como masculinos los atributos de la escala de dureza y como femeninos los de la de ternura, acentuando y polarizando éstas aún más las diferencias percibidas en cuanto a conductas de rol de género. El anclaje sociocultural de los estereotipos de violencia de género tiene implicaciones teóricas y también sociales al visibilizar especialmente el maltrato de hombre a mujer en la pareja heterosexual y desenfocar el que se da en otras formas de pareja. Ello plantea urgencias en la agenda de la investigación.

Palabras clave: violencia, pareja, género, estereotipos.
\end{abstract}

\begin{abstract}
The overall goal of this research was to assess the degree of social attachment of certain stereotypes about gender (male provider; female caregiver) and violence (violent, peaceful woman) and is framed in the context of a debate about the extent and limits of a gender approach when it comes to understanding and preventing violence in different types of partner. 741 people were involved in the research, two thirds of them women, living in Spain, Mexico, Puerto Rico and El Salvador. In each country, they agreed to a stratified convenience sample according to criteria of gender, age, education level, occupational status and sexual orientation. In one session lasting between 35 and 60 minutes, the participants first answered an IAT (Implicit Association Test) and then a series of items in a questionnaire with closed and open ended questions. One section includes 48 items referring to "activities" that the person must categorize numerically on a scale of 1-7, with a semantic differential format, and whose poles are "male" and "woman." In this series two scales of 24 items each are mixed: hardness and tenderness. From the information obtained it is seen that samples from all countries organize their perception of partner violence according to gender stereotypes. Men and women both perceived attributes of the hardness scale to be masculine, and those of tenderness to be feminine, with these perceived differences in terms of gender role behaviors being even more enhanced and further polarized by the women. The socio-cultural anchor of the gender violence stereotype has theoretical and social implications in that it visualizes abuse from a man to a woman in the heterosexual couple and blurs that which occurs in other forms of partner. This raises topics which should be urgently addressed in the research agenda.
\end{abstract}

Key words: violence, couple, gender, stereotypes.

La correspondencia sobre este artículo puede dirigirse a la primera autora a la Universidad Autónoma de Barcelona. Edificio B. Departamento de psicología Social. 08193 Bellaterra-Barcelona. Email: leonor.cantera@uab.es 


\section{Introducción}

Una versión tradicional de la mirada de género, ya bastante arraigada en el sentido común contemporáneo occidental, induce a pensar y a hablar sobre el género sociocultural en los mismos términos dicotómicos del discurso convencional sobre el sexo: en la aparentemente biológica división sexual del trabajo, laten imperativos culturales de género, en función de los cuales el varón se realiza masculinamente como productor y proveedor, cumpliendo su rol agéntico (Deaux, 1985), vertebrando su identidad en el desempeño laboral del rol profesional (job model, Dex, 1988) y su actividad social en la dimensión pública, centrada en la generación de valores de cambio (Blanch, 2003). La hembra humana hace lo propio femeninamente, como reproductora y cuidadora, desempeñando su rol comunal (Deaux, 1985), desarrollándose como mujer en el ámbito privado, ejerciendo el rol doméstico (gender model, Dex, 1988) y en la producción de valores de uso (Blanch, 2003).

El enfoque de género -clave para la comprensión de las relaciones entre hombres y mujeres- facilita la visibilidad teórica y empírica de la violencia del hombre a la mujer en la pareja heterosexual. Por ello viene orientando, desde hace ya algunos lustros, la investigación y la intervención sobre malos tratos en la pareja (Comisión Europea, 2000; Ferreira, 1992; Osborne, 2001; Walker, 1980), hasta el punto en que la misma Ley Orgánica (2004) de medidas de protección integral contra la violencia en la pareja se presenta como la ley contra la violencia de género. Tal enfoque, basado en la premisa de la dualidad de género, define los criterios para una lectura teórica y para la prevención práctica de esta relevante problemática social. Su marcado énfasis heterocentrista induce a centrar de modo casi exclusivo la atención en la violencia que se da en el seno de una pareja heterosexual "normal", en la dirección de hombre a mujer; esto es, en el escenario socialmente correcto de un modelo "patriarcal" de familia (Cantera, 2007).

Esta investigación tiene como objetivo general la evaluación del grado de anclaje social de estereotipos sobre género y sobre violencia de género y parte de la sospecha de que los estereotipos generales sobre género (rol masculino de proveedor; rol femenino de cuidado$r a$ ) y los específicos sobre violencia de género (hombre violento, mujer pacífica) tienen un notable grado de arraigo social en el sentido común, tanto en hombres como en mujeres, y son socialmente relevantes en la medida en que influyen en las explicaciones comunes de las causas de la violencia en la pareja y también en el modo como se piensa la prevención de la misma.

\section{MÉTODO}

\section{Participantes}

Para la puesta a punto de los instrumentos de recogida de información, se accedió a una muestra de con- veniencia integrada por 155 personas españolas, estratificada de acuerdo con criterios de género, generación, nivel de formación, situación ocupacional y orientación sexual. En el trabajo de campo participaron 741 personas, un $65 \%$ de las cuales mujeres, residentes en diversos países del ámbito hispanoamericano (España, México, Puerto Rico y El Salvador), a las que se accedió con los mismos criterios aplicados en la fase piloto.

\section{Procedimiento, instrumentos y técnicas de recogida y análisis de la información}

En sesiones individualizadas, cuya duración osciló entre 35 y 60 minutos, las personas participantes respondieron primero un IAT (Implicit Association Test), en las condiciones ambientales requeridas por el protocolo de la prueba, tecleando directamente un ordenador portátil, dotado del soft correspondiente. A continuación rellenaron un cuestionario de papel y lápiz, que incluye series de ítems cerrados y de preguntas abiertas.

Tradicionalmente, para el estudio empírico de prejuicios y estereotipos han sido utilizadas las más diversas "técnicas explícitas", como tests proyectivos, entrevistas en profundidad, diferencial semántico o escalas de actitudes, cuyo uso conlleva ciertos problemas de control de variables como las "estrategias de autopresentación" o la tendencia a expresarse de acuerdo con el "pensamiento políticamente correcto". En este punto, el Implicit Association Test constituye una innovación metodológica (Nosek, Greenwald \& Banaji, 2005), al estudiar actitudes implícitas, midiendo la fuerza asociativa entre conceptos. Entre las ventajas del uso de esta técnica, destacan la simplicidad de su diseño, la facilidad y rapidez de su uso, su aplicación por ordenador, su aplicabilidad a grupos y su adaptabilidad a múltiples campos temáticos, entre los que destacan los de actitudes y estereotipos (Ortiz y Ruiz, 2004; Aberson y Beeney, 2007).

Según sus diseñadores (Greenwald, McGhee, y Schwartz, 1998), asociaciones fuertes (compatibles) entre pares de concepto diana - atributo (dimensión evaluativa) son más fáciles de clasificar juntas que asociaciones débiles (incompatibles) entre los mismos (Banaji y Greenwald, 1995). Un ejemplo convencional de asociación fuerte (compatible) aportado por Greenwald et al. (1998) es el de flor-agradable vs. insecto-desagradable. Por el contrario la conjunción flor-desagradable vs. insecto- agradable constituye un ejemplo de asociación débil o incompatible. Esta fase incompatible conlleva una mayor demanda cognitiva a la hora de clasificar los diferentes estímulos que irán apareciendo en la pantalla del ordenador. Los resultados del IAT se obtienen a través de los tiempos de respuesta, medidos en milisegundos, y de los errores en las categorizaciones. En general, cuando una persona 
se sitúa en una situación incompatible (flor-desagradable vs. insecto-agradable), tarda más en clasificar palabras como amapola o mosca. En tales casos, se espera un mayor tiempo de respuesta y también una más elevada tasa de errores. Siguiendo con el ejemplo, el efecto IAT se manifiesta en una actitud general más positiva hacia las flores que hacia los insectos. La aplicación del IAT pone empíricamente de relieve lo que ocurre cuando se sustituye el binomio flores e insectos por otros psicológica o socialmente más relevantes.

Para el presente estudio se diseñó un IAT que presenta como categoría-diana hombre-mujer y como atributo violencia-paz. En la fase piloto, se validó la serie de verbos elegidos como estímulos y se controló algunas de las características relevantes de los términos elegidos (tipicidad, familiaridad y frecuencia de uso) que podrían repercutir en el tiempo de reacción (Ortiz y Ruiz, 2007). Se supone que el efecto IAT se manifiesta en la fase "incompatible" (mujer-violencia vs. hombre-paz), que conlleva -para la persona culturalmente normalizada- una mayor demanda cognitiva a la hora de clasificar las diferentes palabras estímulo (como, por ejemplo, conversar o insultar) que aparecen en la pantalla del ordenador, exigiéndole una mayor lentitud en su proceso de clasificación. En tal contexto, se suele producir un mayor tiempo de respuesta y también una mayor tasa de errores. Para el cálculo del IAT D Effect, se aplica el procedimiento algorítmico propuesto por Greenwald, Nosek y Banaji (2003).

El IAT utilizado consta de una fase compatible y de una incompatible con 60 ensayos cada una ellas. La cantidad de estímulos por categoría es de cinco en el caso de violencia (Abusar, Humillar, Dañar, Imponer, Insultar) - paz (Pactar, Escuchar, Respetar, Dialogar, Conversar) y de uno en el caso de hombre - mujer, siendo este estímulo la misma categoría diana. Las palabras - estímulo, elegidas en la fase piloto, atendiendo a criterios como los de su frecuencia de uso en español y su longitud silábica, se repiten aleatoriamente a lo largo de los 60 ensayos practicados de cada fase.

Una de las secciones del cuestionario consiste en una serie de 48 ítems referidos a "actividades" que la persona debe categorizar numéricamente en una escala de 1 a 7 con formato de diferencial semántico en cuyos polos figuran "de hombre" y "de mujer". En estos 48 ítems se entremezclan los correspondientes a dos escalas de 24 ítems cada una de ellas: (a) Los 24 ítems incluidos en la Escala de Dureza (alpha: .90) son los siguientes: Abusar; Acosar; Agredir; Amenazar; Controlar; Chantajear; Dañar; Dominar; Forzar; Golpear; Humillar; Imponer; Insultar; Intimidar; Manipular; Maltratar; Mentir; Obligar; Oprimir; Pegar; Pelear; Provocar y Vulnerar. (b) Por su parte, la Escala de Ternura (alpha: .87) incluye los 24 ítems siguientes: Acariciar; Acordar; Amar; Apoyar; Atender; Colaborar; Compartir; Complacer; Concordar;
Confiar; Conversar; Cooperar; Cuidar; Curar; Dialogar; Empatizar; Escuchar; Gozar; Hablar; Negociar; Proteger; Respetar; Simpatizar y Tolerar.

En otro apartado del cuestionario, se pide la opinión acerca de la cantidad de violencia física (golpes, empujones, patadas, etc.) y psicológica (humillaciones, insultos, amenazas o intimidaciones verbales, chantaje emocional, etc.) que se supone que se da de hombre a mujer y de mujer a hombre en la pareja (heterosexual). Para ello, la persona debe puntuar su estimación en una escala de 1 (Poquísima Violencia) a 7 (Muchísima Violencia). En la serie de preguntas abiertas se invita a buscar explicaciones de tales formas de violencia física y psicológica.

Para el tratamiento estadístico de datos IAT, se usó el soft SuperLab, para el de los datos numéricos del cuestionario el SPSS y para el de los textuales el Atlas $t i$.

\section{Resultados $^{1}$}

En la tabla 1, aparecen las medias y las desviaciones respectivas de las dos condiciones de aplicación en las IAT, en el cual se da una distribución normal $(\mathrm{Z}$ Kolmogorov-Smirnov $=.570)$. Un análisis de la variancia intersujetos mediante One-Way Anova no revela diferencias estadísticamente significativas entre las dos condiciones $(\mathrm{P}=.235)$.

La media total del IAT (.459) indica un alto nivel de anclaje del estereotipo de violencia de género (hombre violento / mujer pacífica) en ambos sexos y en todas las orientaciones sexuales. Sólo un $11 \%$ de participan-

Tabla 1. Estadísticos del IAT según condiciones de aplicación

\begin{tabular}{lcc}
\hline Condiciones & Medias & Desviaciones \\
\hline 1 & .421 & .34 \\
2 & .487 & .33 \\
\hline Total & .459 & .34 \\
\hline
\end{tabular}

tes obtiene puntuaciones negativas en el IAT. El análisis de la variancia revela diferencias estadísticamente significativas al respecto en cuanto el sexo $(\mathrm{p}<.001)$. La media de los hombres es de .26 y la de las mujeres de .55; lo cual indica a su vez que las mujeres de la muestra perciben más intensamente la asociación hombre-violencia y mujer-paz que los hombres de la misma.

De la información extraída de las respuestas al cuestionario de papel y lápiz, aplicado a gente "normal" de

\footnotetext{
1 Obtenidos en el marco de un proyecto subvencionado por el Instituto de la Mujer. exp. 13/05. IP: Leonor M Cantera, UAB.
} 
la calle, se desprende que la mayoría de las personas participantes categoriza las actividades "de hombre" o "de mujer" de acuerdo con los estereotipos de género; esto es, adecuándolas al patrón dicotómico hombremasculino-proveedor-dominador y violento / mujerfemenina-cuidadora-paciente y pacífica. Hombres y mujeres de las muestras de todos los países concuerdan en dar puntuaciones inferiores a 3 (en una escala de 1 a 7 , en la que 1 significa totalmente propio del hombre y 7 totalmente propio de la mujer) a una serie de actividades casualmente revestidas de connotaciones socialmente negativas, como son las de acosar, agredir, golpear, intimidar, maltratar, pegar y torturar. En contrapartida, también coinciden en asignar puntuaciones superiores a 4.6 en la misma escala a una serie de actividades características del rol de género femenino, entre las que destacan las de acariciar, atender, colaborar, conversar, cuidar, curar, dialogar, escuchar, hablar y tolerar, que casualmente están revestidas de connotaciones socialmente positivas.

La tabla 2 presenta estadísticos de las puntuaciones en las escalas de dureza y de ternura diferenciadas por sexo-género, donde se observa que, dentro de esta fundamental coincidencia de puntos de vista entre hombres y mujeres, éstas tienden a acentuar aún más las diferencias en cuanto a conductas de rol de género: una comparación de medias indica que esta serie de actividades de género aparecen significativamente diferenciadas por sexo, de modo que las mujeres tienden a puntuarlas de modo significativamente más polarizado que los hombres en el siguiente sentido: las mujeres valoran como propias de su categoría algunas actividades características del rol de género femenino, asignándoles puntuaciones significativamente más próximas al 7 (polo "de mujer") que las que les dan los hombres. Es lo que se observa a propósito de la escala de ternura. En coherencia con esta visión, las mujeres de la muestra no sólo valoran como propias de los hombres algunas actividades características del rol de género masculino, sino que les asignan puntuaciones significativamente más próximas al 1 (polo "de hombre") que las que les dan los mismos hombres.

Tabla 2. Diferencias en escala DUREZA y TERNURA, según Sexo-Género

\begin{tabular}{lcccc}
\hline ESCALAS & Sexo & Media & Desv. & Sig \\
\hline DUREZA & Hombre & 3,43 &, 64 &, 000 \\
& Mujer & 3,24 &, 61 & \\
\hline TERNURA & Hombre & 4,33 &, 49 &, 000 \\
& Mujer & 4,64 &, 50 & \\
\hline
\end{tabular}

En esta misma línea, los datos obtenidos indican que esta tendencia a la polarización de los roles de género aparece significativamente más marcada en los países latinoamericanos de la muestra, tomados en su conjunto, que en la muestra española en lo que concierne a la acentuación del conjunto de los atributos incluidos en la escala de dureza como más propios del hombre ( $\mathrm{p}<$ $.000)$.

Atendiendo al conjunto de los atributos incluidos en la escala de ternura, aparecen también significativas diferencias entre las muestras por países $(\mathrm{p}<.000)$, siendo las de El Salvador y Puerto Rico las que los puntúan como más propios de la mujer, en contraste con las de México y de España, que presentan una relación algo menos intensa entre aquellos atributos y la condición femenina.

En cuanto a las preguntas del cuestionario donde la persona puntúa (en una escala de 1 a 7 ) la cantidad de violencia "física" o "psicológica" que percibe en una pareja heterosexual (de hombre a mujer y de mujer a hombre), las medias más altas de ambas formas de violencia corresponden a la dirección de hombre a mujer. A ese respecto, la violencia física obtiene una puntuación media total de 4,35 $(1,65)$ y la psicológica de 4,50 $(1,72)$. En contraste, en la dirección de mujer a hombre, la violencia física obtiene una puntuación media total de $2,65(1,32)$ y la psicológica de $3,86(1,52)$. Las diferencias entre ambas direcciones (de hombre a mujer vs de mujer a hombre), tanto en lo que concierne a violencia física como a la psicológica, aparecen como estadísticamente significativas $(\mathrm{p}<.000)$. En la Tabla 3 se presenta las medias (y desviaciones) en violencia (percibida) física y psicológica en la pareja, según sexo.

Tabla 3. Violencia física y psicológica percibida en la pareja, según sexo

Medias (desviaciones)

\begin{tabular}{llcc} 
Violencia & Sexo & Física & Psicológica \\
\hline \multirow{2}{*}{ Hombre $\rightarrow$ Mujer } & Hombre & $4,01(1,59)$ & $4,05(1,69)$ \\
& Mujer & $4,53(1,66)$ & $4,76(1,67)$ \\
& Sig. &, 000 &, 000 \\
\hline & Hombre & $2,71(1,44)$ & $3,90(1,50)$ \\
Mujer $\rightarrow$ Hombre & Mujer & $2,60(1,26)$ & $3,86(1,53)$ \\
& Sig. &, 317 &, 283 \\
\hline
\end{tabular}

Como se observa en la tabla 3 , tanto en lo concerniente a la violencia física como a la psicológica, la dirección hombre-mujer es percibida como aquella en la que se da en mayor medida la violencia. En este marco de coincidencia en lo fundamental, se observa también un significativo contraste entre las respectivas valoraciones de hombres y mujeres, puntuando éstas de modo significativamente más alto que aquéllos tanto la violencia física como psicológica. En cambio, las mujeres de la muestra no se diferencian de los hombres de la misma a la hora de puntuar la cantidad de 
violencia física y la psicológica que perciben en la dirección mujer -hombre en la pareja. Ambos asignan puntuaciones relativamente bajas a la violencia física y unos niveles intermedios a la violencia psicológica.

En lo que concierne a los contrastes entre países, resultan observables las siguientes tendencias: En cuanto a la dirección de hombre a mujer, las muestras de El Salvador y Puerto Rico perciben unos niveles de violencia física y psicológica (con puntuaciones entre $5 \mathrm{y}$ 6 en ambos tipos) más altos que las de España y México (puntuaciones entre 4 y 5 en ambos tipos), apareciendo como estadísticamente significativas $(\mathrm{p}<.000)$ estas diferencias entre países. Algo similar ocurre en lo concerniente a la dirección de mujer a hombre, si bien aquí el promedio baja considerablemente en todos los casos: El Salvador y Puerto Rico dan puntuaciones medias de entre 3 y 4 a la violencia física y de entre 4 y 5 a la psicológica; mientras que España y México puntúan entre 2 y 3 la física y entre 3 y 4 la psicológica.

Ante una serie de preguntas abiertas del cuestionario sobre "motivos" de la violencia en la pareja, se reproduce el consenso básico a la hora de señalar y nombrar una letanía de "causas" (que algunas obras sobre la materia suelen incluir en el listado de los "mitos" en torno a la violencia en la pareja): alcohol, drogas, dificultades económicas y laborales, marginación social y cultural, bajo nivel de instrucción, psicopatología, etc. Asimismo, cuando se pide a las personas encuestadas que cuenten alguna historia que conozcan de cerca sobre violencia en pareja, salen a relucir los mismos tópicos. Y a la pregunta sobre presuntas razones "justificadoras" de violencia en la pareja, la mayoría responde que "nada" la justifica; "pero" que, cuando ocurre, "será" por alguno de los "factores" ya "conocidos": alcohol, drogas, problemas económicos y laborales, infidelidad, celos, carácter, trastornos de personalidad, etc.

\section{Discusión}

Puesto que se está estudiando el grado de arraigo social de estereotipos de género, y en especial de los relacionados con género y violencia, las tendencias generales observadas resultan tan o más relevantes a nivel teórico y social que las eventuales diferencias entre las diversas categorías de cada variable censal considerada. La información obtenida mediante el IAT y la proporcionada por las respuestas numéricas y textuales al cuestionario indican al unísono que la percepción social de las relaciones de pareja se fundamenta en términos generales en los estereotipos de género (hombre-proveedor, mujer-cuidadora) y que la de la violencia en la pareja está filtrada específicamente por el paradigma de violencia de género (hombre-violento, mujer-pacífica). En su conjunto, estos resultados son consistentes con lo esperable desde la tradición teórica y empírica acerca del arraigo social de los estereotipos de género (Ávila, et al., 1995; Campbell, 1993; Comisión Europea, 2000; Deaux, 1985; Dex, 1988; Ferreira, 1992; Gómez, 2003; Osborne, 2001) y sobre la asociación género - violencia (Cantera, 2007; Island y Letellier, 1991; Leventhal y Lundy, 1999; Renzetti y Harvey, 1996; Ristock, 2002; Walker, 1980).

Desde un punto de vista teórico, las informaciones obtenidas respectivamente de la aplicación del IAT y del cuestionario son consistentes y se refuerzan mutuamente. En el plano empírico, destaca por un lado la fundamental coincidencia de los respectivos puntos de vista de hombres y de mujeres a la hora de adoptar una mirada de género y, por otro, la tendencia de éstas a acentuar aún más las diferencias en cuanto a conductas de rol de género, polarizando más las puntuaciones y enfatizando las distancias percibidas entre las conductas típicamente masculinas (atributos incluidos en la escala de dureza) y las consideradas más propias del rol de género femenino (conjunto de los atributos incluidos en la escala de ternura). Esta misma perspectiva reaparece en la valoración de la cantidad percibida de violencia "física" o "psicológica" en una pareja heterosexual (de hombre a mujer y de mujer a hombre), donde la figura del hombre encarna el agente de la violencia y la de la mujer la del sujeto paciente de la misma. También a este respecto las mujeres de la muestra se diferencian de los hombres de la misma al puntuar más alto que aquéllos en la cantidad de violencia física que perciben en la dirección hombre-mujer en la pareja. Tal contraste puede indicar o bien una tendencia de las mujeres a maximizar ciertas características negativas del otro género, para marcar distancias con respecto al mismo, o bien una de los hombres a minimizarlas (por la necesidad psicológica de autoevaluarse positivamente o menos negativamente al respecto) o bien, finalmente, la interacción de ambas tendencias.

De esta percepción social de una mujer naturalmente pacífica y de un hombre propenso a la violencia, anclada en un sentido común conformado por estereotipos de género y de violencia asociada al género, se desprende la premisa implícita, también socialmente compartida, de que la relación de pareja heterosexual (hombre- mujer) conlleva más riesgo de maltrato físico y psicológico que otras modalidades de relación de pareja, que parecerían así más inmunes al mismo (Balsam, 2001; Coleman, 1994; Eaton, 1994; Elliott, 1996; Island y Letellier, 1991; Kaschak, 2001; Ki, 1999; Lobel, 1986; Ohms y Müller, 2001; Ohms y Stehling, 2001; Rystok, 2001, 2002).

\section{Conclusiones}

Los datos obtenidos corroboran la persistente presencia en población hispanoamericana de sesgos y tendencias ya constatados por la literatura científica, especialmente por la anglosajona, sobre el papel desempe- 
ñado por el paradigma género en la percepción social de la violencia en la pareja como violencia de género. Y ponen de manifiesto que el discurso cotidiano sobre violencia en la pareja remite implícitamente al escenario socialmente correcto del sistema patriarcal y a su núcleo duro, configurado por un modelo familia tradicional, organizado en torno a la pareja normal, de carácter heterosexual, cuyas relaciones se rigen por el código de género.

Este anclaje sociocultural de los estereotipos de violencia de género tiene implicaciones teóricas y también sociales: el punto de vista generalizado sobre la violencia normal en la pareja "normal" contribuye -implícita y sutil, pero efectivamente- por una parte, a la visibilización social de este fenómeno problemático $\mathrm{y}$, por otra, a la invisibilización de aquellas formas de violencia en la pareja que no encajan en el paradigma violencia de género. La centralización, focalización y visibilización del maltrato ejercido por un hombre violento sobre una mujer pacífica en una relación de pareja heterosexual comporta, a su vez, cierto grado de desatención, desenfoque e invisibilización de fenómenos y procesos de violencia en una dirección no normal (de mujer a hombre) o en parejas no estándar, como las de tipo gay o lésbico (Elliot, 1990; 1996; Gonsioreck y Weinrich, 1991; Island y Letellier, 1991; Leventhal y Lundy, 1999; Renzetti y Harvey, 1996; Ristock, 2002). Estas "otras" modalidades de relación de pareja comportan, a su vez, otras posibles causas y sentidos de la violencia, ante las cuales constructos como los de "patriarcado" o "machismo" acaso aporten más ruido y confusión que clarificación de la problemática.

Lo que es poco visible y menos pensable y explicable teóricamente a los ojos del sentido común resulta aún más difícil de prevenir en la práctica. Ello conlleva nuevos e importantes desafíos para la investigación y también para la intervención sobre una problemática como la de las víctimas del maltrato en relaciones de pareja homosexual -gay y lésbica- con especiales dificultades para nombrar, pensar, comprender y comunicar su propio proceso y para ser reconocidas y atendidas como tales por la sociedad y por sus instituciones.

En la agenda de la investigación figura analizar en qué medida y en qué sentido los estereotipos sociales sobre violencia de género encontrados en la gente de la calle están también anclados entre profesionales del derecho, de la policía y de las ciencias humanas, sociales y de la salud; por la relevancia de su implicación en la identificación, en el reconocimiento, en la previsión y en la prevención de la problemática de la violencia en cualquier tipo de relación de pareja.

\section{Referencias}

Aberson, C. L. y Beeney, J. (2007). Does substance use affect reliabilities of the Implicit Association Test? The Journal of Social Psychology, 147, 27-40.
Ávila, M. Cruz, E., Fernández, E., B.; González, J. J., Román, F. A. y Schmidt, I (1995). La más casera de las violencias sociales: violencia contra la pareja. San Juan, Puerto Rico. De una orilla a otra orilla

Balsam, K. (2001). Nowhere to hide: Lesbian battering, homophobia, and minority stress. En Kaschack, E. (Eds.). (2001). Intimate betrayal: Domestic violence in lesbian relationships. (pp. 25-37). New York: The Haworth.

Banaji, M. R. y Greenwald, A. G. (1995). Implicit gender stereotyping in judgments of fame. Journal of Personality and Social Psychology, 68, 181-198.

Blanch, J.M. (2003). Trabajar en la modernidad industrial. (13-148). En J.M. Blanch (Coord). Teoría de las Relaciones Laborales. Vol I. Fundamentos. Barcelona: Editorial UOC.

Brown, C. (2008). Gender-Role Implications on Same-Sex Intimate Partner Abuse. Journal of Family Violence, 23, 457-462.

Campbell, A. (1993). Men, women and aggression. New York: Basic Books.

Cantera, L. (2007). Violência no casal. Um enfoque além do gênero. Porto Alegre, Brasil: Dom Quixote.

Coleman, V. (1994). Lesbian battering. The relationship between personality and the perpetration of violence. Violence and Victims, 9, 139-152.

Comisión Europea (2000). Romper el silencio. Campaña europea contra la violencia doméstica. Luxembourg: Oficina de Publicaciones Oficiales de las Comunidades Europeas.

Deaux, K. (1985). Sex and gender. Annual Review of Psychology, 36, 4981.

Dex, S. (1988). Women's attitudes towards work. London. MacMillan.

Eaton, M. (1994). Abuse by any other name: Feminism, difference, and intralesbian violence. En M. Albertson y R. Mykitiuk, (Eds.), The public nature of private violence: The discovery of domestic abuse. (pp. 195-224). New York: Routledge.

Elliott, P. (Ed.). (1990). Confronting lesbian battering: A manual for the battered women's movement. St. Paul: Minnesota coalition for battered women.

Elliott, P. (1996). Shattering illusions: Same sex domestic violence. En Renzetti, C. y Harvey, C. (Eds.). Violence in gay and lesbian domestic partnerships. (pp. 1- 8). New York: Harrington Park.

Ferreira, G. (1992). Hombres violentos. Mujeres maltratadas. Aportes a la investigación y tratamiento de un problema social. Buenos Aires: Sudamericana.

Gómez, V. (2003). No seré feliz, pero tengo marido. Madrid: Temas de hoy.

Gonsioreck. J. C. y Weinrich, J. D. (1991). Homosexuality: Research Implications for Public Policy. Newbury Park (CA): Sage.

Greenwald, A. G., McGhee, D. E., \& Schwartz, J. L. K. (1998). Measuring individual differences in implicit cognition: The Implicit Association Test. Journal of Personality and Social Psychology, 74, $1464-1480$.

Greenwald, A. G., Nosek, B. A. y Banaji, M. R. (2003). 
Understanding and using the Implicit Association Test: I. An improved scoring algorithm. Journal of Personality and Social Psychology, 85, 197-216.

Island, D. y Letellier, P. (1991). Men who beat the men who love them. New York: Routledge.

Kaschak, E. (Ed.). (2001). Intimate betrayal. Domestic violence in lesbian relationships. New York: The Haworth.

Ki (1999). Sharing my story. En Leventhal, B. y Lundy, S. (Eds.). Same-sex domestic violence. Strategies for change. (pp. 3-7). Newbury Park, Cal: Sage.

Leventhal, B. y Lundy, S. (Eds.). (1999). Same-sex domestic violence. Strategies for change. Newbury Park, Cal: Sage.

Ley Orgánica (2004). Ley orgánica de medidas de protección integral contra la violencia de género. Madrid: Jefatura del Estado. Ley Orgánica 1/2004 de 28 de diciembre.

Lobel, K. (Ed.). (1986). Naming the Violence: Speaking Out About Lesbian Battering. Seattle, WA.: Seal.

Nosek, B.A., Greenwald, A.G. y Banaji, M.R. (2005).Understanding and Using the Implicit Association Test: II. Method Variables and Construct Validity. Personality and Social Psychology Bulletin. 31, 166-180.

Nosek, B. A., Greenwald, A. G. y Banaji, M. R. (2007). The Implicit Association Test at age 7: A methodological and conceptual review (pp. 265-292). In J. A. Bargh (Ed.), Automatic processes in social thinking and behavior. Washington: Psychology Press.

Ohms, C. y Müller, K. (2001). In good hands? The status quo of psycho-social assistance for lesbian victims of violence and/or discrimination: A European comparison. Frankfurt: AntiViolence Project LIBS.

Ohms, C. \& Stehling, K. (2001). Violence against lesbiansViolence against gay men: Thesis on Differences and Similaritis. First European Symposium: Violence against Lesbians. (190-222). Berlin: Querverlag GmbH.

Ortiz, M. y Ruiz, M. (2004). Áreas de aplicación del IAT. Revista de Psicología Social Aplicada, 14, 31-48.

Ortiz, M. y Ruiz, M. (2007). Facilitando la aplicación del "Implicit Association Test" (IAT): una demostración práctica en estudios sobre violencia y género. $\mathrm{X}$ Congreso Nacional de Psicología Social: 26 - 28, IX. Cádiz. Actas.

Osborne, R. (ed.). (2001). La violencia contra las mujeres. Realidad social y políticas públicas. Madrid: UNED.

Renzetti, C. y Harvey, C. (Eds.). (1996). Violence in gay and lesbian domestic partnerships. New York: Harrington Park.

Ristock, J. (2002). No more secrets. Violence in lesbian relationship. New York: Routledge.

Ristock, J. (2001). Decentering heterosexuality: Responses of feminist counselors to abuse in lesbian relationships. In Kaschack, E. (Ed.). (2001). Intimate betrayal: Domestic violence in lesbian relationships. (pp. 59-72). New York: The Haworth.

Walker, L. (1980). The battered woman. New York: Harper Perennial.

Manuscrito Recibido: 08/01/2010

Revisión Recibida: 26/03/2010

Manuscrito Aceptado: 26/03/2010 